International Journal of

Astrobiology

\section{cambridge.org/ija}

\section{Letter}

Cite this article: Edwards MR (2021). Ectogenesis for survival in deep space and deep time: reply to Gale and Wandel. International Journal of Astrobiology 20, 252-253. https://doi.org/10.1017/ S1473550421000094

Received: 25 February 2021 Revised: 4 March 2021 Accepted: 8 March 2021

First published online: 29 March 2021

Author for correspondence: Matthew R. Edwards, E-mail: matt.edwards@utoronto.ca

C The Author(s), 2021. Published by Cambridge University Press

\title{
Ectogenesis for survival in deep space and deep time: reply to Gale and Wandel
}

Matthew R. Edwards (D)

University of Toronto, John P. Robarts Library, 6th Floor, Toronto, Ontario, Canada M5S 1A5
Gale and Wandel (2021) describe my paper in IJA (Edwards, 2021) as having crossed the border of scientific plausibility into the realm of 'science fiction.' By science fiction, they presumably mean the 'bad' kind based on implausible or unsupported scientific concepts, such as time reversal, multiverses and indeed the warp drives of Star Trek they mention. As my paper is based only on current medical and scientific research, it does not make the transgression they imply. In it, I first reviewed artificial uterus systems and embryo cryopreservation as to whether they could potentially be used in space colonization or in recolonizing Earth after mass extinction events. While complete ectogenesis - the development of early-stage embryos to birth entirely outside the natural womb - is not yet available to serve this purpose, in the near future it likely will be (Bulletti et al., 2011; Räsänen and Smajdor, 2020). To illustrate the power and flexibility of the approach, I then discussed how such systems might be deployed in a comprehensive survival plan to handle a variety of extinction events, ranging from brief events in the very near future to the final, total extinction due to solar expansion (Ward and Brownlee, 2003; Klee, 2017).

Gale and Wandel direct their criticisms primarily at these example survival missions, not the feasibility of ectogenesis with cryopreserved embryos per se. On one hand, they argue that near-term extinction events could be better handled by establishing colonies on Mars, on Jupiter's moons or in O'Neill-type space colonies. Such colonies have dim prospects indeed. Recently it was shown, for example, that insufficient $\mathrm{CO}_{2}$ exists in known reservoirs on Mars to enable terraforming (Jakosky and Edwards, 2018), a sine qua non for Martian colonies. Jupiter's moon Europa has indeed been proposed as a possible candidate for a space colony (e.g., the Artemis Project), but the extremely harsh radiation environment due to Jupiter's magnetic storms and the ultra-frigid surface conditions there render such a colony as pure fantasy. Gerard O'Neill's space colonies - city-sized structures holding up to a million people - rely on such notions as harvesting raw materials for manufacturing from the Moon or asteroids and constructing a totally self-supporting internal ecosystem. Such achievements again go far beyond what is technically feasible in the foreseeable future. Moreover, if by some miracle such colonies were in fact built, it would be Earth which would have to continually sustain them, rather than they protecting us from possible extinction. After a major extinction event, the vital connection with Earth for everything from medicines to spare parts would be lost and the colony would quickly collapse.

In the model scheme I proposed, however, smaller spacecraft or space stations orbiting the Earth and carrying just a small number of astronauts/colonists would be one of the first lines of defence in short-duration events. These missions could then be extended using ectogenesis to handle events of progressively larger magnitude and longer duration, by substituting embryos first for some and then finally all of the adult crew members. These orbiting missions together with an interlocking, parallel sequence unfolding in subterranean installations would be the simplest and also the most urgent application of ectogenesis to mass extinction survival, a process I termed Embryo Earth Recolonization (EER).

While these EER missions would be the most critical ones in terms of human survival, Gale and Wandel save most of their fire for the far more technically challenging missions of exoplanet colonization, previously termed Embryo Space Colonization (ESC). These missions would be needed to avoid extinction events that render Earth permanently inhospitable to life, including the final one due to solar expansion. They first argue that it would be pointless to send out ESC spacecraft now, with existing methods of propulsion, as they would only be overtaken by later generations of spaceships with faster propulsion. In the first place, missions to exoplanets would most likely not be undertaken until many years of experience had first been gained through designing EER missions for mass extinction survival. By that time significant advances in propulsion systems would presumably have been made. More significantly, an advanced civilization that repeatedly delayed sending out ESC ships could run the risk of being obliterated in an extinction event before any ships were launched.

On a deeper level, whether the ships are faster or slower does not really matter. Gale and Wandel's argument that the mechanical systems on ESC ships could not survive for thousands or millions of years would be true: if the systems were running that whole time. As I 
emphasized, however, these ships would need to be seed-like themselves, remaining in an electronically and mechanically dormant state for almost the entire interstellar journey. Whether the periods of dormancy last for thousands of years or millions in this case makes little difference. Coasting at ship temperatures close to absolute zero and with suitable radiation shielding, there is no theoretical reason to suppose that the ship's electronic systems, machinery - and embryos - could not survive these epic transits.

The problems encountered at the exoplanet would indeed be enormous, as Gale and Wandel point out, but the pathways to solving them are all laid out in my paper. (Most of these were addressed in the section on far-future Earth recolonization missions, for which the problems are very similar.) Whereas manned missions would face immediate and intense survival pressure at an exoplanet, ESC ships could adopt a leisurely approach. A succession of modules carrying species would establish ecosystems in an ordered sequence, each stage lasting many years. The human modules would land on the planet surface only after functioning food chains had already been built up. Even if the cryonic suspension of astronauts should become feasible - and progress here lags far behind that of ectogenesis - Gale and Wandel's preference for it to colonize exoplanets is also questionable. Do they propose that a large supporting population of farm animals be sent in cryonic suspension also? And what would those animals feed on? At a lifeless exoplanet, it would be technically far simpler for androids and AI to build up supporting ecosystems and food chains from scratch, using frozen microbes, plant seeds and animal embryos. Cryonically suspended astronauts might then land on the terraformed world, but a better solution would be to raise human colonists from embryos too.

I did not propose, as Gale and Wandel imply, that embryo missions 'would solve the civilization longevity problem in Drake's equation,' only that they could vastly increase the value of that Drake term. Embryo missions would likely be the most feasible means of securing long-term survival for advanced civilizations generally, as it logically holds that the adults of other intelligent species would also typically develop from an embryo stage. As the puzzle of life's origin has not been solved, there is no conflict with Fermi's Paradox, as they stated. However, the embryo missions do suggest a place where one might look for advanced civilizations. Since mass extinction events would be frequent on all Earthlike planets, by definition, the survivors might be found in embryonic form, perhaps in orbiting spacecraft or subterranean bunkers.

To sum up, Gale and Wandel are incorrect in stating that survival missions involving ectogenesis and cryopreserved embryos cross over into the realm of scientific implausibility. Techniques for partial ectogenesis will be available for clinical use in just a few years and ones for complete ectogenesis quite likely soon after. Embryo missions would indeed face steep challenges, but the science behind them is sound. That is fortunate indeed, for they may be needed for the long-term survival of humanity and human civilization.

Conflict of interest. None.

\section{References}

Bulletti C, Palagiano A, Pace C, Cerni A, Borini A and de Ziegler D (2011) The artificial womb. Annals of the New York Academy of Sciences 1221, 124-128.

Edwards MR (2021) Android Noahs and embryo Arks: ectogenesis in global catastrophe survival and space colonization. International Journal of Astrobiology 20, 150-158.

Gale G and Wandel A (2021) On the border between science and science fiction. International Journal of Astrobiology, 20, in press.

Jakosky BM and Edwards CS (2018) Inventory of $\mathrm{CO}_{2}$ available for terraforming Mars. Nature Astronomy 2, 634-639.

Klee F (2017) Human expunction. International Journal of Astrobiology 16, $379-388$.

Räsänen J and Smajdor A (2020) The ethics of ectogenesis. Bioethics 324, 328-330.

Ward PD and Brownlee D (2003) The Life and Death of Planet Earth: How the new Science of Astrobiology Charts the Ultimate Fate of our World. London: Piatkus. 